\title{
Components of Mathematics Anxiety: Factor Modeling of the MARS30-Brief
}

\author{
Belinda Pletzer ${ }^{*}$, Guilherme Wood ${ }^{1,2}$, Thomas Scherndl1', Hubert H. Kerschbaum ${ }^{3}$ and \\ Hans-Christoph Nuerk ${ }^{4}$ \\ 1 Department of Psychology, Paris-Lodron University Salzburg, Salzburg, Austria, ${ }^{2}$ Department of Psychology, University of \\ Graz, Graz, Austria, ${ }^{3}$ Department of Cell Biology, Paris-Lodron-University Salzburg, Salzburg, Austria, ${ }^{4}$ Department of \\ Psychology, Eberhard Karls University Tübingen, Tübingen, Germany
}

Mathematics anxiety involves feelings of tension, discomfort, high arousal, and physiological reactivity interfering with number manipulation and mathematical problem solving. Several factor analytic models indicate that mathematics anxiety is rather a multidimensional than unique construct. However, the factor structure of mathematics anxiety has not been fully clarified by now. This issue shall be addressed in the current study. The Mathematics Anxiety Rating Scale (MARS) is a reliable measure of mathematics anxiety (Richardson and Suinn, 1972), for which several reduced forms have been developed. Most recently, a shortened version of the MARS (MARS30-brief)

OPEN ACCESS

Edited by:

Carlo Tomasetto

University of Bologna, Italy

Reviewed by:

Prathiba Natesan,

University North Texas, USA

Caterina Primi

University of Florence, Italy

*Correspondence:

Belinda Pletzer

belinda.pletzer@sbg.ac.at

Specialty section:

This article was submitted to

Cognition,

a section of the journal

Frontiers in Psychology

Received: 29 July 2015

Accepted: 15 January 2016

Published: 17 February 2016

Citation:

Pletzer B, Wood G, Scherndl T, Kerschbaum $\mathrm{HH}$ and Nuerk $\mathrm{H}-\mathrm{C}$ (2016) Components of Mathematics Anxiety: Factor Modeling of the MARS30-Brief. Front. Psychol. 7:91. doi: 10.3389/fpsyg.2016.00091 with comparable reliability was published. Different studies suggest that mathematics anxiety involves up to seven different factors. Here we examined the factor structure of the MARS30-brief by means of confirmatory factor analysis. The best model fit was obtained by a six-factor model, dismembering the known two general factors "Mathematical Test Anxiety" (MTA) and "Numerical Anxiety" (NA) in three factors each. However, a more parsimonious 5-factor model with two sub-factors for MTA and three for NA fitted the data comparably well. Factors were differentially susceptible to sex differences and differences between majors. Measurement invariance for sex was established.

Keywords: mathematics anxiety, confirmatory factor analysis, Mathematics Anxiety Rating Scale, sex differences, career choice

\section{INTRODUCTION}

High arousal and physiological reactivity in response to number manipulation are symptoms of mathematics anxiety (Richardson and Suinn, 1972; Dew et al., 1984; Faust, unpublished doctoral dissertation). They lead to avoidance of careers that require mathematical skills (Ashcraft and Faust, 1994; Ashcraft and Kirk, 2001; Hopko et al., 2001). Accordingly, women typically have higher values of mathematics anxiety than men (e.g., Devine et al., 2012) and mathematics anxiety differs across college majors (e.g., Preston, 1986, but see Hamza et al., 2011). However, mathematics anxiety may therefore contribute to impaired life functioning (e.g., Hopko et al., 2001).

Therefore quick and efficient identification of mathematics anxious persons by standardized instruments is important for intervention (see Richardson and Suinn, 1973 for an intervention study). For application in adults Richardson and Suinn (1972) constructed a measure of anxiety 
related to mathematics originally consisting of 98 items - the Mathematics Anxiety Rating Scale (MARS), which has been validated by several studies (Richardson and Suinn, 1972, 1973; Suinn et al., 1972; Brush, 1978; Morris et al., 1978). Since then, several studies developed abbreviated forms in order to reduce administration time or eliminate contaminated items that did not fit data (69 and 25 items by Alexander and Martray, 1989; 10 items by Ferguson, 1986; 34 items by Fujii, 1994; 12 items by Hopko, 2003; 67 items by Levitt and Hutton, 1984; 24 items by Plake and Parker, 1982; 94 items by Rounds and Hendel, 1980; see also Table 1). Summarizing results of Rounds and Hendel (1980), Alexander and Cobb (1987) and Alexander and Martray (1989), the authors of the original instrument themselves constructed a shortened scale consisting of 30 items, called the MARS30brief (Suinn and Winston, 2003). They report a Cronbach's alpha of 0.96 and test-retest reliabilty of 0.90 for this instrument and consider it to be comparable to the original 98 item scale.

Several studies tried to disclose the factor structure of mathematics anxiety by applying factor analyses to different versions of the MARS. Table 1 gives an overview of the factor structures obtained with different extraction methods, different samples, and test versions. Generally, a global 2-factor-structure is widely accepted (Rounds and Hendel, 1980; Alexander and Cobb, 1987). Different authors distinguish between two aspects of mathematics anxiety: "Mathematics Test Anxiety" (MTA) describing anxiety associated with learning for mathematics tests and being evaluated in mathematics, and "Numerical Anxiety" (NA) describing anxiety associated with the manipulation of numbers, basic arithmetic skills, and monetary decisions in everyday situations (see Rounds and Hendel, 1980; Alexander and Cobb, 1987).

Since mathematics test-related items evoke more anxiety than task- or course-related items (Alexander and Martray, 1989) some authors consider MTA to be the more important factor of mathematics anxiety and NA to play only a secondary role (Plake and Parker, 1982; Alexander and Martray, 1989). Therefore, Plake and Parker (1982) developed the MARS-R, which consists only of items concerning the MTA-factor. However, these authors still tried to base their measure on a multilevel model of mathematics anxiety and take into account that it is related to general state-, trait-, and test-anxiety. These authors described 2 subscales of the MARS-R or MTA: "Learning Mathematics Anxiety" (LMA), concerning learning for mathematics tests or homework, and "Mathematics Evaluation Anxiety" (EA), concerning mathematics tests and exams. This structure has been validated and replicated through confirmatory factor analysis by Hopko (2003). Interestingly, Alexander and Cobb (1987) assign a subset of items categorized as "Course Anxiety" to the MTAfactor, which are considered to be part of the NA scale by other authors (see Table 1). In summary, most studies report a one or two-factor structure of the MTA scale.

Regarding the factor structure of the global dimension NA, studies reveal a more fine-grained factor structure. The NA-factor is subdivided into "Everyday Numerical Anxiety" (ENA, Bessant, 1995), "Performance Anxiety" (PA, Bessant, 1995), "Social Responsibility Anxiety" (SRA, Resnick et al., 1982), "Observation Anxiety" (OA, Bessant, 1995), and "Problem Solving Anxiety"

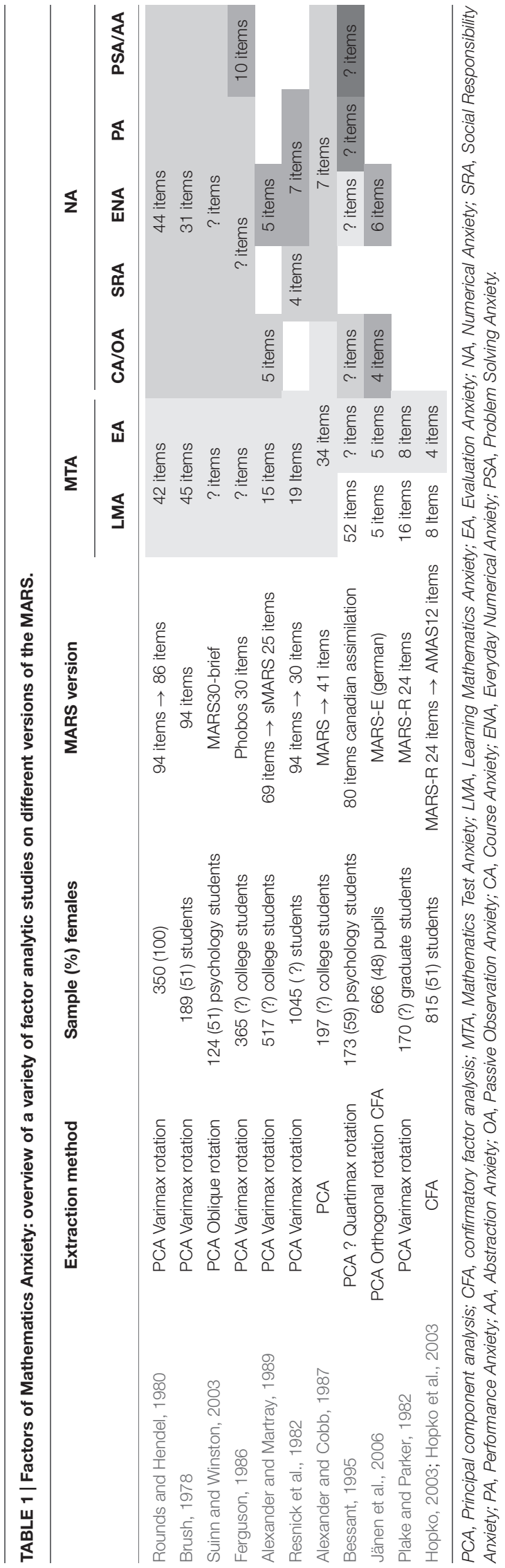


(PSA, Bessant, 1995), or "Abstraction Anxiety" (AA, Ferguson, 1986). ENA involves private calculations in everyday situations, while PA includes performance pressure induced by being told to solve mathematical problems. SRA concerns everyday life situations demanding social responsibility, e.g., memorizing figures for a driving license test. OA involves watching someone working on mathematical problems, with a calculator or on the blackboard. PSA/AA concerns abstract mathematical problem solving like equations or ratios. As can be depicted from Table 1 a great variety of factor solutions has been obtained for the items pertaining to the NA scale.

In summary, the factor structure of mathematics anxiety remains unclear. Different reasons for this may be pointed out: In part this can be attributed to the large diversity of (i) extraction methods and (ii) item sets employed, and (iii) assignment of items to factors.

(i) First, a great variety of methods employed to investigate the covariance structure of the MARS can be observed (Table 1). While most authors have worked with exploratory methods for determining the number of factors necessary for accounting for a substantial proportion of variance (principal components analysis with different rotation methods, scree plot, fixation of the number of factors), only one study has so far used confirmatory factor analysis to investigate whether the MTA-factor consisted of one or two subfactors. On the one hand, exploratory methods imply dangers concerning overfactorization in the final item selection (Fabrigar et al., 1999). For instance, when the average item covariance is relatively low, the exploratory solution may reveal too many factors. On the other hand, relevant portions of the covariance structure of the original items set may be overseen when many items were eliminated, because they load on two or more separate factors simultaneously.

(ii) Secondly, the item selection in the different studies differed widely and was often not even explicitly reported. Some studies obtained their abbreviated versions not from the original 98items scale, but from non-validated abbreviated item subsets. For instance, Rounds and Hendel (1980) ran a factor analysis over 94 out of the 98 original items, while Bessant (1995) used only 80 items and Alexander and Martray (1989) 69 items. Furthermore, Ferguson (1986) used 20 items that according to Rounds and Hendel (1980) loaded on one of the two factors MTA and NA, as well as 10 further items referring to abstract mathematical topics. The MARS-R of Plake and Parker (1982) consisted mainly of Items of the MTA-factor and, according to the authors, was designed for application in "statistically related situations" (Plake and Parker, 1982, p. 552). Problems with the lack of selection criteria may cumulate over studies when authors develop new reduced versions of the MARS from abbreviated item sets taken from the literature (Resnick et al., 1982; Hopko, 2003). As a result, the factor structure of abbreviated versions of the MARS may tap on very specific subset of the dimensions described in the literature (Table 1). To summarize, the widely varying item selections for different factor analyses may have led to very differing empirical and theoretical factor solution. In particular, some reduced version of the MARS may ignore important dimensions of mathematics anxiety and may be useful only for investigating specific aspects of this construct.
(iii)Third, the assignment of items to factors as described in the literature is very often incomplete. While, Plake and Parker (1982), Ferguson (1986), Alexander and Martray (1989), and Hopko (2003) reported exactly the assignment of all items surviving factor analysis to their respective factors as well as their loads in these factors, other authors have reported the assignment of items to factors only in an illustrative way. Therefore, it is possible that some items may have been assigned to different factors over different studies. Once more the unclear assignment of items to determined factors may lead to problems with the conceptual interpretation of the different dimensions of mathematics anxiety.

For these reasons further investigation on the factor structure of mathematics anxiety is still necessary. Specifically, it is relevant to determine (i) whether the traditional two-factor model by Rounds and Hendel (1980) is sufficient for describing the dimensionality of mathematics anxiety, (ii) whether these two factors as second-order factors can be dismembered into several smaller first-factors in a hierarchical CFA model and (iii) whether the second-order factors are necessary for describing the dimensionality of mathematics anxiety. In the present study we therefore examined and compared these three confirmatory factor analytic models. Especially, the MARS is probably still the most widely used mathematics anxiety questionnaire and the MARS30-brief is its present (abbreviated) version. While Richardson and Suinn (1972) report an internal consistency of 0.97 and a test-retest reliability of 0.85 for the MARS, Suinn and Winston (2003) report an internal consistency of 0.96 and test-retest reliability of 0.90 for the MARS-30 brief. According to the authors, validity data also support the comparability of the two measures. Thus, the MARS30-brief can be considered an economical equivalent of earlier versions of the MARS, which has been constructed under consideration of results from earlier studies, also accounting for their deficiencies in selection of samples and item sets. Therefore, disclosing its factor structure is of great empirical interest. To our knowledge, the factor structure of the current version of this diagnostic instrument has not been investigated with confirmatory factor analytic techniques yet. Therefore, in the present study the factor structure of the MARS30-brief was examined.

Establishing the factor structure of mathematics anxiety may help identifying, which aspects of the construct lead to the avoidance of careers requiring mathematical skills. When considering MTA and NA, it is of interest, whether the anxiety pertains to the performance of mathematics in itself, irrespective of the situation, or whether the anxiety is more strongly attributed to the test situation. The present study aims to evaluate, whether more sub-factors are necessary to gain an even closer picture of where and when the anxiety manifests for an individual. In particular it may be relevant, whether it already leads to the avoidance of learning math (LMA) or only to the avoidance of test situations (EA) or whether it leads to the avoidance of performing math in everyday life altogether (ENA) or only in situations of social responsibility (SRA).

Identifying, which aspects of math anxiety are most important for a person, is, however, of importance for successful intervention. Therefore, in the present study, after establishing 
the factor structure of the MARS30-brief, we will also assess individual differences in these sub-factors, particularly gender differences and differences between college majors. While gender differences and differences between college majors are commonly accepted for mathematics anxiety, only few studies have so far distinguished between different components of mathematics anxiety in these comparisons. This may in part be attributable to the fact that inconsistencies already arise, when taking only the two factors MTA and NA into account. According to Evans (2000) higher values in women were confirmed for both MTA and NA, whereas Baloğlu and Koçak (2006) report higher MTA values in women, but higher NA values in men using a revised version of the MARS. Furthermore, it has been suggested based on different relationships of MTA and NA to age and attitudes toward mathematics in men and women that the factor structure of the mathematics anxiety may differ between men and women (Wilder, 2012). This has, however, not been confirmed using confirmatory factor analytic models. Therefore, we will establish measurement invariance prior to our gender comparisons, while the comparisons between majors need to remain exploratory due to small sample sizes in some groups. However, to the best of our knowledge, it has not been previously investigated, whether gender differences and differences across college majors, concern all sub-factors of MTA and NA or whether some factors are more sensitive for gender- and major-differences than others.

\section{MATERIALS AND METHODS}

\section{Participants}

Participants were 491 students (330 women, 161 men, mean age: 21.78 years, $S D=4.05$ years; range: $18-55$ years) at the University of Salzburg. 162 of the participants (96 women, 66 men) were enrolled as psychology majors, 179 (124 women, 55 men) were enrolled as biology majors, 46 (26 women, 20 men) were enrolled as mathematics majors and 66 (55 women, 11 men) were enrolled as language majors. The remaining 38 participants were from other majors (e.g., education, history, geography) or did not provide any information about their major. The latter were not included in analyses comparing mathematics anxiety between majors.

\section{Ethics Statement}

Participants were informed about the aims of the study and gave a written consent authorizing data processing for research purposes. Participation in the present study was voluntary. To assure anonymity in data processing, a numerical code was assigned to each participant. All methods conform to the Code of Ethics of the World Medical Association (Declaration of Helsinki).

The institutional guidelines of the University of Salzburg (Statutes of the University of Salzburg see http://www.uni-salzburg.at/fileadmin/multimedia/Senat/ documents/Satzung.pdf) state in $\$ 163$ (1) that ethical approval is necessary for research on human subjects if it affects the physical or psychological integrity, the right for privacy or other important rights or interests of the subjects or their dependents.
In $\$ 163,(2)$ it is stated that it is the responsibility of the PI to decide, whether (1) applies to a study or not. Therefore we did not seek ethical approval for this study. Since it was non-invasive and performed on healthy adult volunteers who gave their informed consent to participate, (1) did not apply.

\section{Measure}

The MARS30-brief was developed by Suinn and Winston (2003) and is a 30-item instrument for individual or groupadministration. Items represent mathematics-related situations that may cause anxiety in the respondent. The translation into German was conducted by the first author and corrected by her supervisors for administration in German-speaking participants (see Table 2 for item examples). Participants reported their level of anxiety associated with a particular item by checking the corresponding token in a scale from "not at all" (0), "a little" (1), "a fair amount" (2), "much" (3) to "very much" (4). Therefore, scores in the individual items ranged from 0 to 4 . The MARS30brief was administered in an auditorium of the University of Salzburg to all participants at once. Measure instructions were read aloud by an experimenter; the same instructions were also printed on the first page of the MARS30-brief's booklet. Instructing and administering the MARS30-brief took a total time of approximately $10 \mathrm{~min}$. One and only one answer for each item was allowed. All participants conformed to these instructions - there were no missing data.

\section{Analyses}

To determine the factor structure of the MARS30-brief, a series of confirmatory factor models was calculated. We started the confirmatory factor analysis by examining the fit obtained for a default model (Model 0) for comparison, including only one global factor for mathematics anxiety (MARS). The first test model (Model 1) included two global factors, named "Mathematical Test Anxiety" (MTA) and "Numerical Anxiety" (NA). The assignment of items to factors MTA and NA was based on that reported by Rounds and Hendel (1980): We assigned items 1-15, all mentioning a mathematics test or exam to MTA, and items 16-30, all mentioning performing mathematics in everyday life to NA (see Figure 1). In a second model (Model 2), the factors MTA and NA were defined as second order factors (Marsh and Hocevar, 1985). Moreover, the first order factors EA and LMA were assigned to the second order factor MTA while the first order factors ENA, SRA, and PA loaded on the second order factor NA (see Figure 2). The assignment of items to EA, LMA, ENA, SRA, and PA was done as described in the literature (see Introduction and Table 1). All items referring to taking a mathematics examination were assigned to EA, all items referring to learning for a mathematics examination to LMA. All items referring to performing mathematics in everyday life (calculating a budget, reading a receipt) were assigned to ENA, all items referring to performing mathematics in a socially responsible role were assigned to SRA and all items simply referring to performing mathematics without giving a context (adding or dividing numbers on a paper) were assigned to PA. Item examples for each factor are listed in Table 2. The full list of items can be found in Suinn and Winston (2003). 


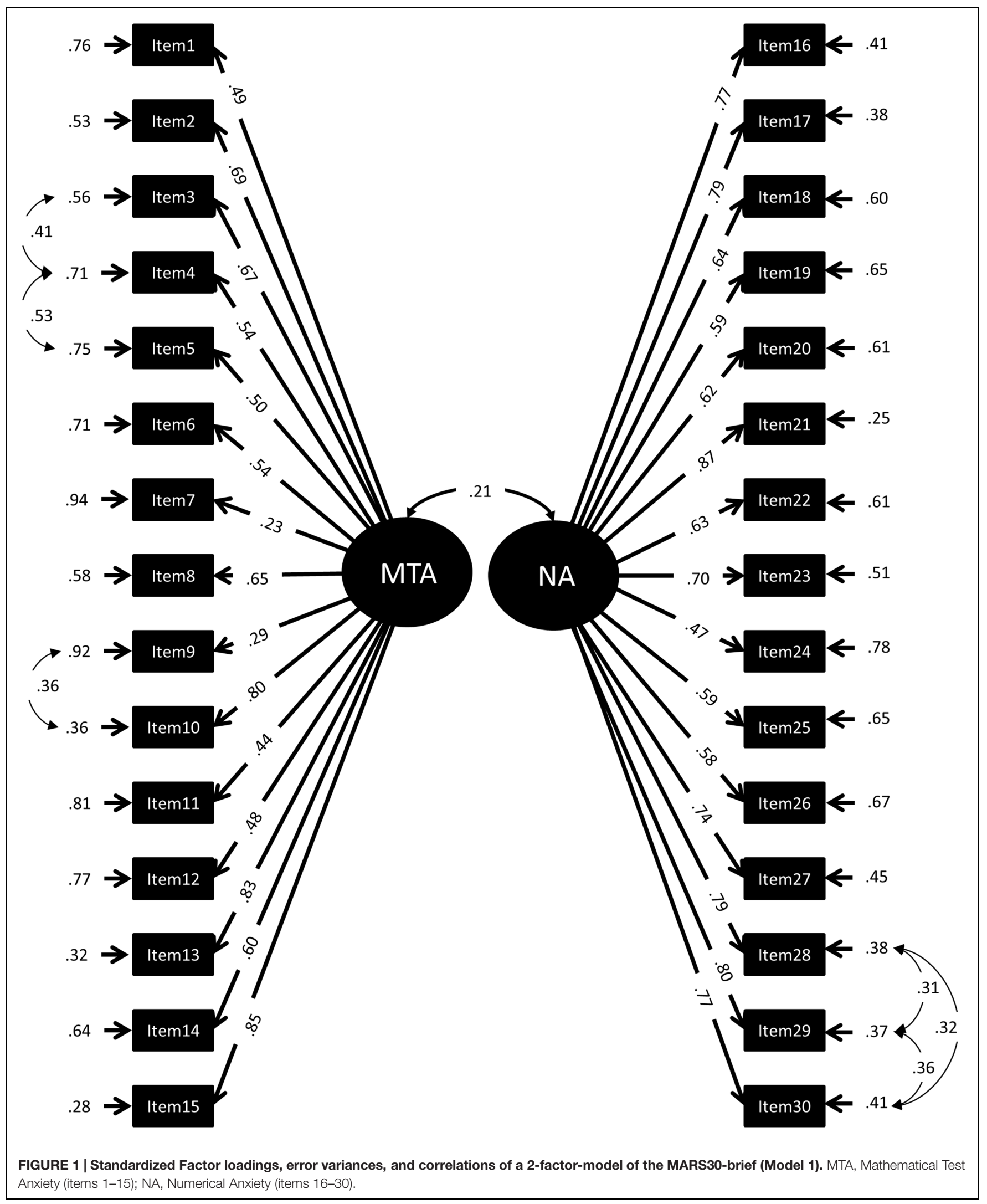




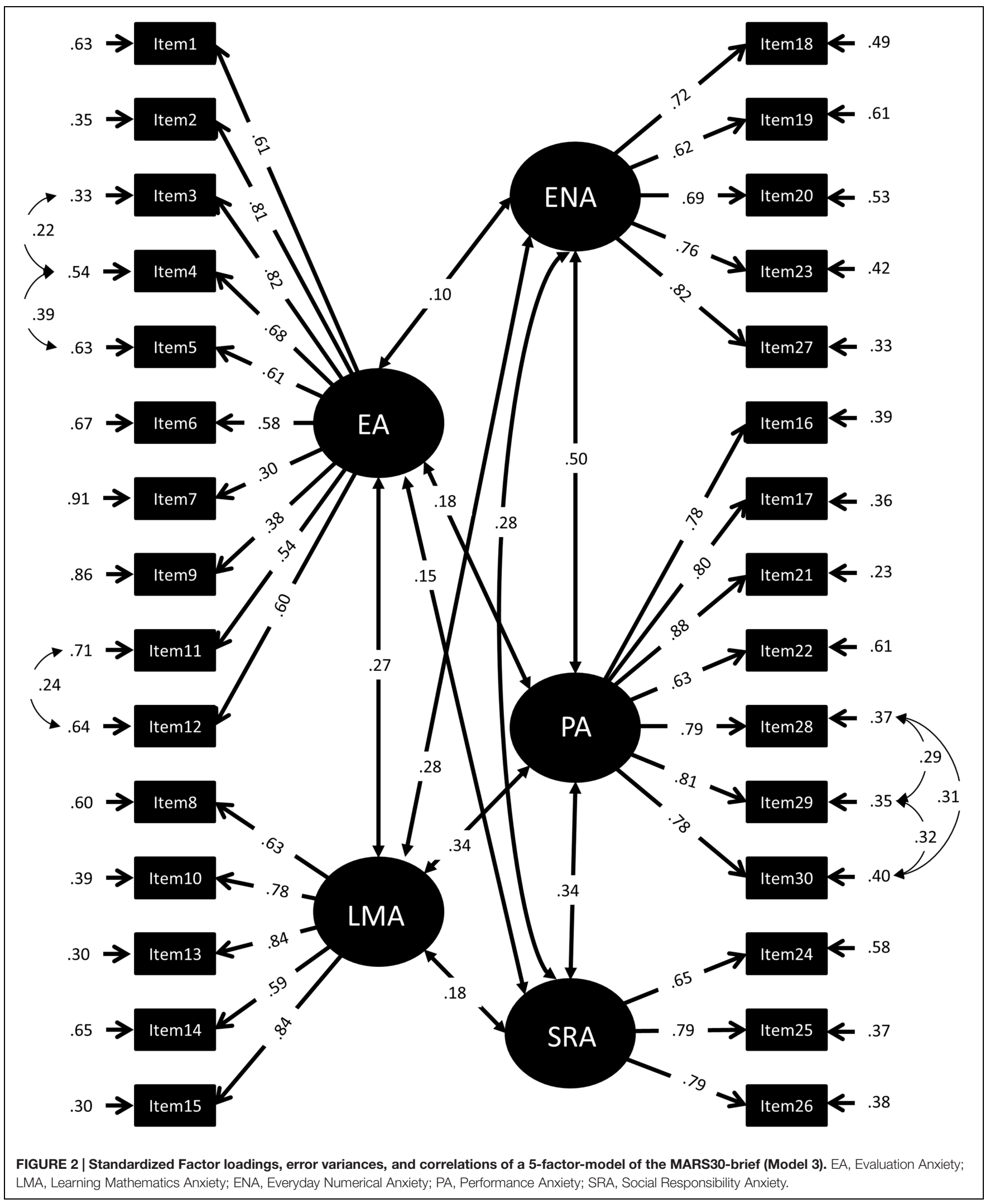


TABLE 2 | Item examples for each factor.

\begin{tabular}{lll}
\hline EA1 & Item1 & $\begin{array}{l}\text { Taking an examination (final) in a mathematics } \\
\text { course }\end{array}$ \\
EA2 & Item4 & $\begin{array}{l}\text { Thinking of an upcoming mathematics test on hour } \\
\text { before. }\end{array}$ \\
LMA & Item10 & $\begin{array}{l}\text { Studying for a mathematics test. } \\
\text { ENA }\end{array}$ \\
PA & Item20 & $\begin{array}{l}\text { Figuring out your monthly budget. } \\
\text { Being given a set of numerical problems involving } \\
\text { addition to solve on paper. }\end{array}$ \\
SRA & Item24 & $\begin{array}{l}\text { Being responsible for collecting dues for an } \\
\text { organization and keeping track of the amount. }\end{array}$
\end{tabular}

a Copyright for the MARS test and all exemplary MARS items is owned by Richard M. Suinn, Ph.D., 808 Cheyenne Drive, Ft. Collins, CO 80525, USA. All rights reserved.

Note that Item 27 mentioned watching others work with a calculator, which would normally be assigned to OA. However, since this was the only item of this kind, it was assigned to ENA. The third model preserved only the first order factors of Model 2 but removed the second order factors (Model 3, see Figure 3).

These 3 Models were constructed following strictly the description of Factors in the literature (compare Table 1). However, we realized that Items 2-6, albeit mentioning a mathematics exam or test, did not refer to actually taking that exam, but to thinking about the exam. In order to test, whether thinking about an examination represented a different component of MTA than actually taking an examination, a fourth model was tested including six instead of five first order factors (Model 4, compare Figure 4). Model 4 included the same factors as Model 3, with the exception that EA was split into EA1, being EA proper (taking an examination) and EA2 (thinking about an examination).

The same correlations between error terms were allowed in each model for items 3-5 (thinking about a mathematics examination a day/hour/minutes before it takes place) and items 28-30 (being supposed to perform divisions/additions/multiplications), because their wording was very similar, in fact differed only in one word.

Since we observed gender differences on some factor scores, but not others, we additionally tested the comparability of each model between men and women. First, model fit was obtained for each group. Then measurement invariance was established. Since total sample size is larger than 300 , strict criteria were used for measurement invariance analysis as recommended in Chen (2007). Measurement invariance for loadings and residuals was assumed, if the reduction in CFI did not exceed 0.01 and the reduction in RMSEA did not exceed 0.015 .

Model estimation and comparison as well as tests for multivariate normality were carried out using the lavaan package for R. To evaluate Model fit we chose the Comparative Fit Index (CFI), since we want to compare the fit between different models, the Tucker-Luis index TLI as a relative fit index, which is not affected by sample size and does penalize adding additional parameters to the model and the Root Mean Square Error of approximation (RMSEA) as a badness of fit index that takes model complexity into account. Models were accepted, if CFI was $>0.95$. Further statistical analyses were carried out using the software SPSS version 20. In particular, subfactor scores were compared to each other using Wilcoxon and Friedman-tests. The total MARS score and the sub-factor scores were compared non-parametrically between genders using Mann-Whitney $U$ tests and between majors using KruskalWallis and Mann-Whitney $U$ tests. For Mann-Whitney $U$ tests between majors, the significance level was Bonferonni-corrected to 0.008 .

\section{RESULTS}

\section{Normative Data}

Participants reached an average total score of 36.83 (SD = 15.69, range: 1- 90). Ordinal alpha (based on the polychoric correlations) was 0.93 . While this was lower than in the initial study of Suinn and Winston (2003) ( $\alpha=0.96)$, it can be considered satisfactory. A significant Kolmogorov-Smirnov test suggested that the MARS30-brief total score deviated from a normal distribution in the present study $(Z=1.46, p=0.03)$. Average responses and standard deviations as well as ordinal alpha with this item deleted are presented for each item in Table 3. As can be depicted from Table 3, deletion of items does not change the reliability of the scale.

\section{Confirmatory Factor Analytic Models}

The covariance structure presented by the 30 items of the MARS30-brief did not follow a multivariate normal distribution based on Mardias test for multivariate normality $\left(X^{2}=384.55\right.$, $p<0.001)$ since neither multivariate skewness $\left(\beta_{1}=207.87\right.$; $\left.X^{2}=17010.56, p<0.001\right)$ nor multivariate kurtosis $\left(\beta_{2}=1347.63 ; Z=98.01, p<0.001\right)$ were within an acceptable range. As indicated by significant Kolmogorov-Smirnov tests, each items deviated from a univariate normal distribution as well $(p<0.001)$. For this reason the CFA-model including all 30 items have been estimated with the unweighted least squares method of estimation (Bentler and Dudgeon, 1996; Schumacker and Lomax, 2004). Since ordinal data were obtained on a Likert scale, CFA-models were based on the polychoric correlation matrix and asymptotic covariance matrix.

In a first step, we evaluated the fit of the default model, with all items assigned to one factor MARS (Model 0). This model did not obtain a satisfactory model fit (compare Table 4), indicating that mathematics anxiety as assessed with the MARS30-brief is comprised of more than one factor.

In a second step different factor structures were tested and compared to the default model. To examine the twofactor structure reported by Rounds and Hendel (1980), we assigned items 1-15 all mentioning a mathematics test or exam to MTA and items 16-30 to NA (Model 1, Figure 1). The high $X^{2}$ value and borderline fit indices associated with Model 1 point out that this two-factor model cannot account for the covariance structure of data satisfactorily (Table 4). This suggests that the structure of mathematics anxiety is more fine-grained than a simple distinction of MTA and NA constructs. Importantly, however, the sum of scores for 


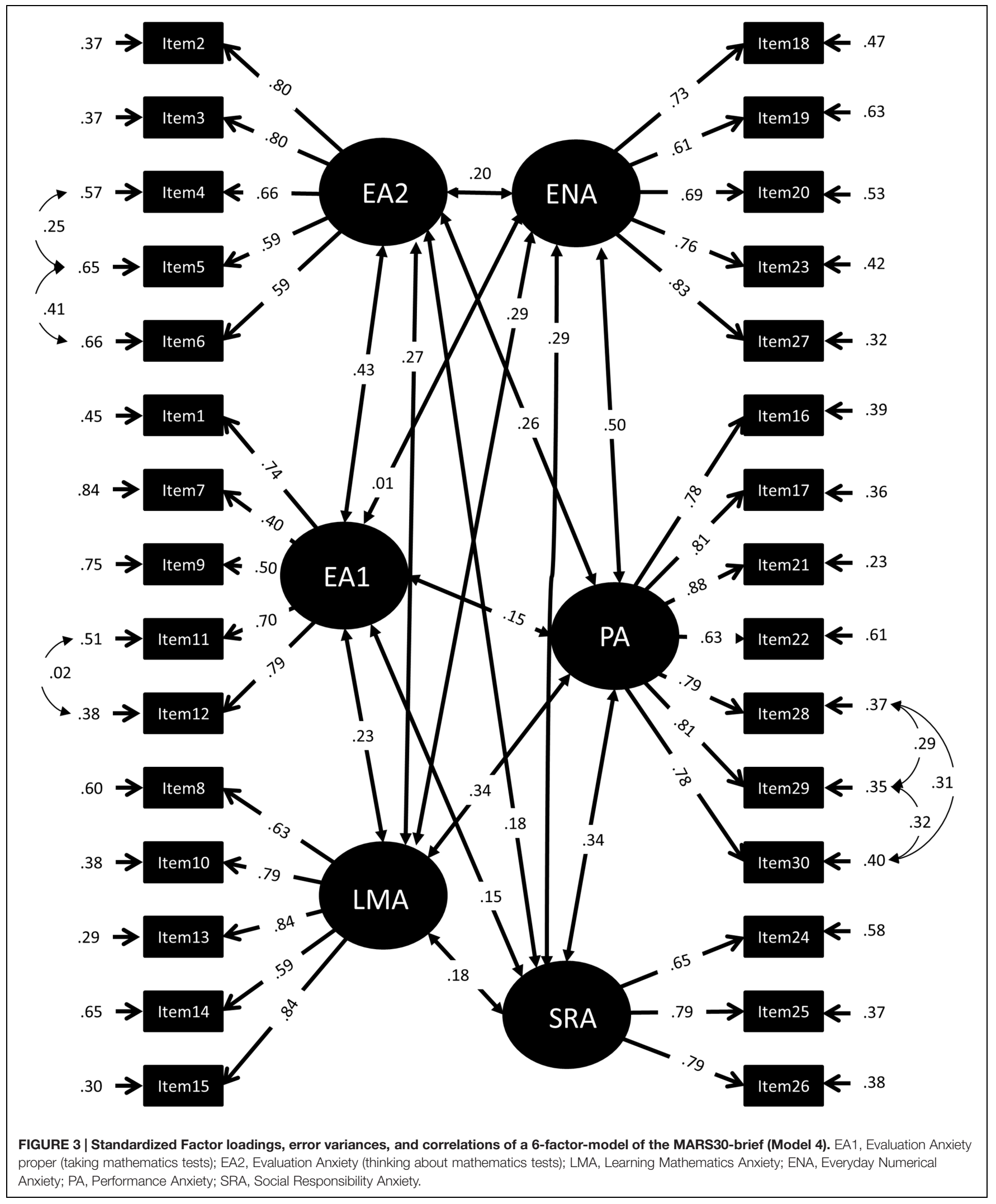




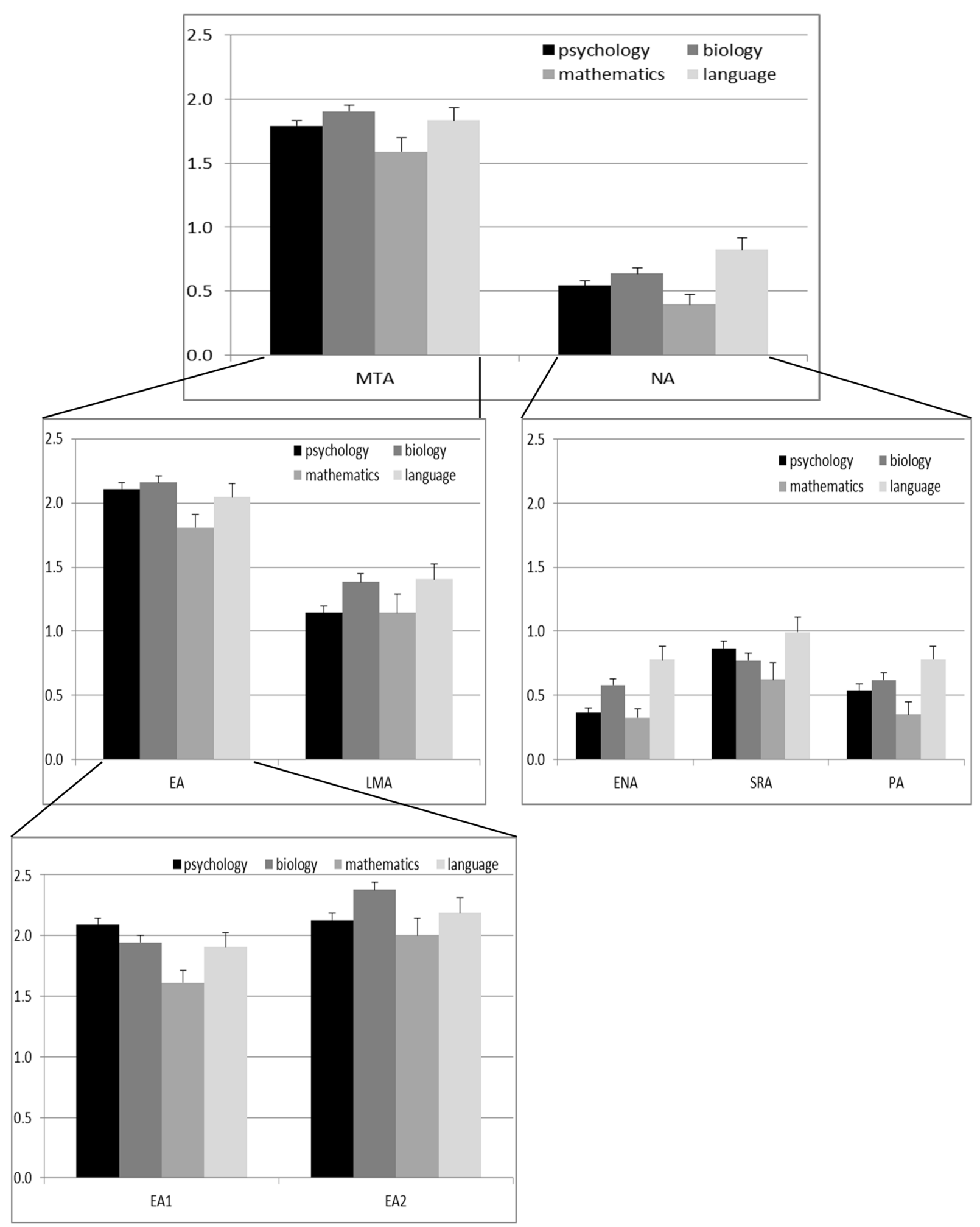

FIGURE 4 | Means \pm SE for the different factors of mathematics anxiety split by major. MTA, Mathematical Test Anxiety (items 1-15); NA, Numerical Anxiety (items 16-30). EA, Evaluation Anxiety; LMA, Learning Mathematics Anxiety; ENA, Everyday Numerical Anxiety; PA, Performance Anxiety; SRA, Social Responsibility Anxiety; EA1, Evaluation Anxiety proper (taking mathematics tests); EA2, Evaluation Anxiety (thinking about mathematics tests). 
TABLE 3 | Item statistics.

\begin{tabular}{|c|c|c|c|c|c|c|c|c|c|c|c|}
\hline & Mean & $S D$ & $\alpha$ if deleted & Skew & Kurtosis & & Mean & $S D$ & $\alpha$ if deleted & Skew & Kurtosis \\
\hline Item 1 & 2.17 & 1.09 & 0.93 & -0.16 & -0.59 & Item 16 & 0.53 & 0.98 & 0.93 & 1.95 & 3.09 \\
\hline Item 2 & 1.74 & 1.08 & 0.93 & 0.17 & -0.54 & Item 17 & 0.37 & 0.89 & 0.93 & 2.60 & 6.07 \\
\hline Item 3 & 2.58 & 1.14 & 0.93 & -0.47 & -0.65 & Item 18 & 0.44 & 0.99 & 0.93 & 2.37 & 4.77 \\
\hline Item 4 & 2.73 & 1.12 & 0.93 & -0.60 & -0.42 & Item 19 & 0.42 & 0.77 & 0.93 & 2.05 & 4.14 \\
\hline Item 5 & 2.75 & 1.17 & 0.93 & -0.60 & -0.54 & Item 20 & 0.75 & 1.07 & 0.93 & 1.44 & 1.28 \\
\hline Item 6 & 1.42 & 1.13 & 0.93 & 0.49 & -0.65 & Item 21 & 0.47 & 0.83 & 0.93 & 1.88 & 3.10 \\
\hline Item 7 & 1.55 & 1.18 & 0.93 & 0.27 & -0.83 & Item 22 & 0.98 & 1.07 & 0.93 & 0.96 & 0.14 \\
\hline Item 8 & 1.65 & 1.23 & 0.93 & 0.27 & -0.90 & Item 23 & 0.73 & 0.94 & 0.93 & 1.26 & 1.13 \\
\hline Item 9 & 2.45 & 1.16 & 0.93 & -0.48 & -0.51 & Item 24 & 1.02 & 1.01 & 0.93 & 0.83 & 0.09 \\
\hline Item 10 & 1.35 & 0.96 & 0.93 & 0.54 & -0.38 & Item 25 & 0.76 & 0.93 & 0.93 & 1.14 & 0.72 \\
\hline Item 11 & 1.91 & 0.96 & 0.93 & -0.11 & -0.86 & Item 26 & 0.75 & 0.90 & 0.93 & 1.10 & 0.67 \\
\hline Item 12 & 1.90 & 1.05 & 0.93 & -0.16 & -0.21 & Item 27 & 0.23 & 0.64 & 0.93 & 3.27 & 11.54 \\
\hline Item 13 & 0.70 & 1.04 & 0.93 & 1.36 & 1.20 & Item 28 & 0.75 & 0.96 & 0.93 & 1.21 & 0.81 \\
\hline Item 14 & 1.53 & 0.98 & 0.93 & 0.30 & -0.50 & Item 29 & 0.53 & 0.84 & 0.93 & 1.71 & 2.73 \\
\hline Item 15 & 1.07 & 0.89 & 0.93 & 0.80 & 0.05 & Item 30 & 0.58 & 0.90 & 0.93 & 1.60 & 2.08 \\
\hline
\end{tabular}

TABLE $4 \mid X^{2}$ and fit indices for the reported models.

\begin{tabular}{|c|c|c|c|c|c|c|c|c|c|c|}
\hline & \multicolumn{5}{|c|}{ Model fit } & \multicolumn{4}{|c|}{ Model comparison } & \\
\hline & $D f$ & $x^{2}$ & CFI & TLI & RMSEA & Model & $\Delta X^{2}$ & $\Delta \mathbf{d f}$ & $P$ & \\
\hline Model 0 (1 Factor) & 399 & 4034.19 & 0.87 & 0.86 & 0.14 & & & & & Reject \\
\hline Model 1 (2 Factors) & 398 & 2471.85 & 0.93 & 0.92 & 0.10 & To Model 0 & 1562.30 & 1 & $<0.001$ & Reject \\
\hline Model 2 (2-stages) & 393 & 1702.98 & 0.95 & 0.95 & 0.08 & \multicolumn{5}{|c|}{ Discarded due to negative error variances } \\
\hline Model 3 (5 Factors) & 389 & 1617.09 & 0.96 & 0.95 & 0.08 & To Model 1 & 854.75 & 4 & $<0.001$ & Accept \\
\hline Model 4 (6 Factors) & 384 & 1373.70 & 0.97 & 0.96 & 0.07 & To Model 3 & 243.39 & 5 & $<0.001$ & Accept \\
\hline
\end{tabular}

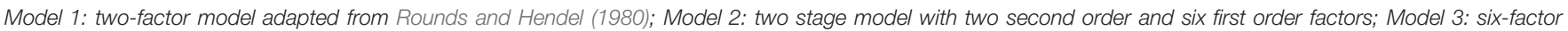
model with no second order factors. CFI, Comparative Fit Index; TLI, Tucker-Lewis Index; RMSEA, Root Mean Square Error of Approximation, for all models $p<0.05$.

TABLE 5 | Factor pattern and structure coefficients for Models 3 (5 Factor) and 4 (6 Factor).

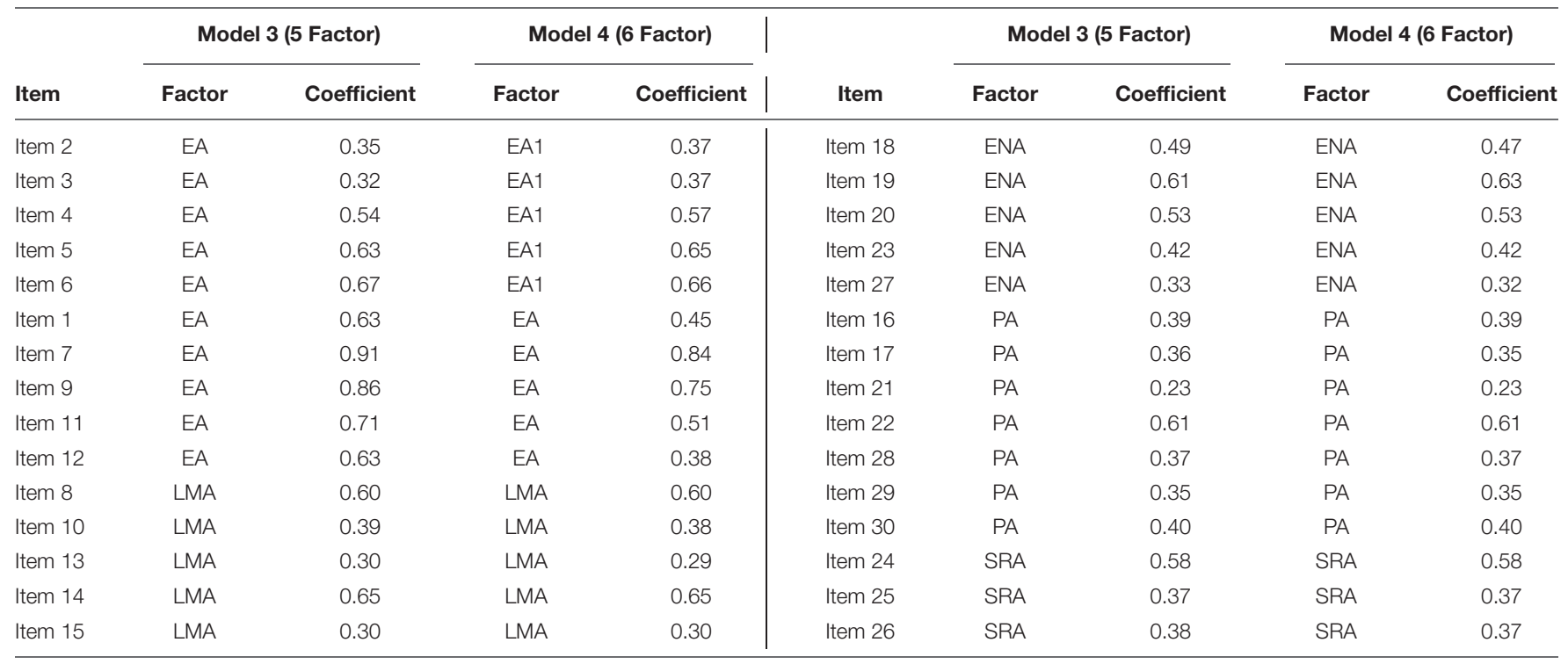

EA, Evaluation Anxiety; EA1, Evaluation Anxiety proper (taking an examination); EA2, thinking about an examination; LMA, Learning Math Anxiety; ENA, Everyday Numerical Anxiety; PA, Performance Anxiety; SRA, Social Responsibility Anxiety. 
TABLE 6 | Latent Factor correlations for Model 3 (5 Factors, below diagonal) and Model 4 (6 Factors, above diagonal).

\begin{tabular}{|c|c|c|c|c|c|c|c|}
\hline & & \multicolumn{2}{|c|}{ EA } & \multirow[t]{2}{*}{ LMA } & \multirow[t]{2}{*}{ ENA } & \multirow[t]{2}{*}{ PA } & \multirow[t]{2}{*}{ SRA } \\
\hline & & EA1 & EA2 & & & & \\
\hline \multirow[t]{6}{*}{ EA } & EA1 & & 0.43 & 0.23 & 0.01 & 0.15 & 0.15 \\
\hline & EA2 & & & 0.27 & 0.20 & 0.26 & 0.18 \\
\hline & & & & & 0.29 & 0.34 & 0.18 \\
\hline & & & & 0.28 & & 0.50 & 0.29 \\
\hline & & & & 0.34 & 0.50 & & 0.34 \\
\hline & & & & 0.18 & 0.28 & 0.34 & \\
\hline
\end{tabular}

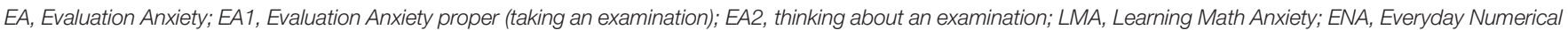
Anxiety; PA, Performance Anxiety; SRA, Social Responsibility Anxiety.

TABLE 7 | Measurement invariance between men and women for Model 3 (5 Factor).

\begin{tabular}{|c|c|c|c|c|c|c|c|c|c|c|c|}
\hline & \multicolumn{4}{|c|}{ Model fit } & \multicolumn{7}{|c|}{ Model comparison } \\
\hline & Df & $x^{2}$ & CFI & RMSEA & Model & $\Delta X^{2}$ & $\Delta \mathbf{d f}$ & $\Delta \mathrm{CFI}$ & $\triangle$ RMSEA & $\boldsymbol{P}$ & \\
\hline Configural & 778 & 2042.60 & 0.96 & 0.08 & & & & & & & Accept \\
\hline Loadings & 803 & 2197.60 & 0.95 & 0.08 & To configural & 154.94 & 25 & $<0.01$ & $<0.015$ & $<0.001$ & Accept \\
\hline Intercepts/residuals & 888 & 2401.60 & 0.95 & 0.08 & To loadings & 204.01 & 85 & $<0.01$ & $<0.015$ & $<0.001$ & Accept \\
\hline Means & 893 & 2894.30 & 0.93 & 0.10 & To residuals & 492.74 & 6 & $>0.01$ & $>0.015$ & $<0.001$ & Reject \\
\hline
\end{tabular}

TABLE 8 | Measurement invariance between men and women for Model 4 (6 Factor).

\begin{tabular}{|c|c|c|c|c|c|c|c|c|c|c|c|}
\hline & \multicolumn{4}{|c|}{ Model fit } & \multicolumn{7}{|c|}{ Model comparison } \\
\hline & Df & $x^{2}$ & CFI & RMSEA & Model & $\Delta X 2$ & $\Delta$ df & $\Delta \mathrm{CFI}$ & $\Delta$ RMSEA & $P$ & \\
\hline Configural & 768 & 1787.50 & 0.97 & 0.07 & & & & & & & Accept \\
\hline Loadings & 792 & 1904.60 & 0.96 & 0.08 & To configural & 117.16 & 24 & $<0.01$ & $<0.015$ & $<0.001$ & Accept \\
\hline Intercepts/residuals & 876 & 2133.40 & 0.96 & 0.08 & To loadings & 228.74 & 84 & $<0.01$ & $<0.015$ & $<0.001$ & Accept \\
\hline Means & 882 & 2632.00 & 0.94 & 0.09 & To residuals & 498.67 & 6 & $>0.01$ & $=0.013$ & $<0.001$ & Reject \\
\hline
\end{tabular}

MTA (Items 1-15; $27.50 \pm 9.85$ ) were significantly higher than the sum or scores for NA (items 16-30; $9.33 \pm 8.81$; $Z=18.75 ; p<0.001)$. Ordinal alphas of MTA and NA were both 0.89 .

In Model 2 the two-factor structure was dismembered into a hierarchical CFA structure with the two original MTA and NA factors as second order factors. To second order factor MTA the first order factors EA and LMA were assigned and to the second order factor NA the first order factors ENA, PA, and SRA. This model resulted in negative error variances, suggesting a bad fit for the data and was therefore discarded. Therefore, Model 3 included only the five factors EA, LMA, ENA, PA, and SRA, but the second order factors MTA and NA were removed (Figure 2). The $X^{2}$ value associated with Model 3 was significantly lower than that of Models 1 and model fit was much better. This suggests that a non-hierarchical five-factor model describes the factor structure of the MARS30-brief better than the two-factor solution in Model 1. Ordinal alphas of the 5 factors in the CFA-model were $0.86,0.86,0.84,0.89$ and 0.96 for EA, LMA, ENA, SRA, and PA, respectively. Average scores for EA $(2.11 \pm 0.71)$ were significantly higher than for LMA $(1.26 \pm 0.81 ; Z=16.75, p<0.001)$. Furthermore, the scores on the sub-factors of NA did differ significantly from each other as indicated by a Friedman test $\left(X^{2}=99.57, p<0.001\right)$. As indicated by Wilcoxon comparisons (all $Z>4.16$, all $p<0.001$ ), ENA $(0.52 \pm 0.03)$ was significantly lower than PA $(0.60 \pm 0.04)$ and SRA $(0.84 \pm 0.04)$, while SRA was significantly higher than PA and ENA.

Furthermore we tested, whether model fit could be further improved, by dismembering the EA factor into EA1 (taking an examination) and EA2 (thinking about an examination), which has not been described in the literature before (Figure 3). Indeed, the model fit obtained by this model (Model 4) was best and the $X^{2}$ value was significantly lower than in Model 3. This suggests that other than described in the literature the MTA factor was comprised of more than two components, since taking an examination and thinking about a examination comprised different sub-factors of MTA. Ordinal alphas of EA1 and EA2 were 0.73 and 0.83 , respectively. Average scores for EA1 (1.99 \pm 0.78$)$ were significantly higher than average scores for EA2 $(2.25 \pm 0.85 ; Z=5.85$, $p<0.001)$.

Model comparisons are also displayed in Table 4 indicating that Model fit was significantly improved in each step. Tables 5 and 6 provide the factor pattern, coefficients and factor correlations for Models 3 and 4. 


\section{Gender Differences}

An analysis of measurement invariance was conducted on Models 3 and 4 to see whether the same factor structure can be obtained for men and women. First, Models 3 and 4 provided comparably good fit for both the male (Model 3: $X^{2}=726.10, \mathrm{df}=389, \mathrm{CFI}=0.96, \mathrm{TLI}=0.96, \mathrm{RMSEA}=0.07$; Model 4: $X^{2}=676.65, \mathrm{df}=384$, CFI $=0.97$, TLI $=0.96$, RMSEA $=0.07$ ) and female subsample (Model 3: $X^{2}=1316.53$, $\mathrm{df}=389$, CFI $=0.95$, TLI $=0.95$, RMSEA $=0.09$; Model 4: $X^{2}=1110.81, \mathrm{df}=384, \mathrm{CFI}=0.96, \mathrm{TLI}=0.96$, RMSEA $=0.07)$. Results for different types of measurement invariance are displayed in Tables 7 and 8. While each additional constraint significantly reduced the $X^{2}$ value of the model, model fit remained acceptable until the last step. Thus, mean factor scores can be compared between men and women.

As described in the literature, the MARS total score was significantly higher in women $(38.48 \pm 15.67)$ than in men $(33.42 \pm 15.22)(Z=3.18, p=0.001)$. Gender differences were only observed in the first 15 items (MTA; $Z=4.40, p<0.001$ ), but not in the second 15 items (NA; $Z=0.33, p=0.74$ ). Gender differences were furthermore confirmed for all sub-factors of MTA (LMA, EA, EA1, and EA2; all $Z>2.48$, all $p<0.05$ ), but only for the sub-factor PA of NA $(Z=1.97, p<0.05)$, not for ENA and SRA (both $Z<0.79$, both $p>0.43$ ).

\section{Differences Between Majors}

Due to small sample sizes in some subgroups analyses of measurement invariance across majors could not be conducted. Therefore the following results are exploratory.

The MARS total score differed significantly between major subjects $\left(X^{2}=15.70, p=0.001\right)$. Mathematics majors had significantly lower values than biology and language majors (all $Z>3.15$, all $p<0.002$ ). Psychology majors had by trend higher values than mathematics majors $(Z=2.32, p=0.02)$, but by trend lower values than biology or language majors (both $Z>1.96$, both $p<0.05$ ). Biology and German majors had comparable values $(Z=0.55, p=0.58)$. Major subject had a significant impact on both MTA and NA. However, Mann-Whitney $U$ tests indicated that while for MTA highest scores were obtained by biology majors (significantly higher than mathematics majors, $Z=2.74, p=0.002$ ), for NA highest scores were obtained by language majors (significantly higher than psychology and mathematics majors, $Z=3.79$, $p<0.001$; compare Figure 4). Significant differences between the majors were also observed for all sub-factors of MTA and NA (all $X^{2}>8.64$, all $\left.p<0.05\right)$. Interestingly, for both EA and LMA the highest scores were obtained by biology majors. However, when split between EA1 and EA2, the highest scores for EA1 were obtained by psychology majors (significantly higher than mathematics majors, $Z=4.07, p<0.001$; not different from biology majors, $Z=1.57, p=0.12$ ), whereas only for EA2 the highest scores were obtained by biology majors (significantly higher than psychology majors, $Z=3.06, p=0.002$ ). For ENA, PA, and SRA, however, the highest scores were obtained by language majors.

\section{DISCUSSION}

As can be depicted from Table 1 a great variety of factor solutions of mathematics anxiety exists. A global two-factor structure consisting of MTA and NA is widely accepted (Rounds and Hendel, 1980; Alexander and Cobb, 1987). However several studies report different sets of smaller factors. In the present study four factor analytic models were carried out in order to disclose the factor structure of mathematics anxiety, in particular the MARS30-brief. We wanted to determine (i) whether the traditional two-factor structure (i.e., MTA and NA as first order factors), first described by Rounds and Hendel (1980) is sufficient for describing the dimensionality of mathematics anxiety, (ii) whether MTA and NA can be dismembered into the first-order factors EA, LMA, ENA, PA, and SRA in a hierarchical CFA model and (iii) whether MTA and NA are necessary for describing the dimensionality of mathematics anxiety and (iv) whether EA could be further subdivided into EA1 (taking mathematics examinations) and EA2 (thinking about mathematics examinations). Furthermore, the present study aimed to evaluate, whether gender differences and differences across majors were comparable across all factors of mathematics anxiety and whether as a consequence the factor structure was comparable between men and women.

Our confirmatory factor models showed that (i) the twofactor structure was only borderline acceptable as description of the MARS30-brief in a single model, (ii) a hierarchical CFA factor structure having MTA and NA as second order factors described data equally well as the non-hierarchical five-factor model including EA, LMA, ENA, PA, and SRA. However, the best fit was obtained for a model including the six first order factors EA1, EA2, LMA, ENA, PA, and SRA. In the following these results will be discussed in more detail. Contrary to previous studies (Evans, 2000; Baloğlu and Koçak, 2006), gender differences with higher scores in women were observed only for MTA, not for NA, however, equally for all sub-factors of MTA (EA, EA1, EA2, and LMA). These differences were, however, not attributable to differences in the factor structure of the MARS between men and women, since measurement invariance for gender could be established. Differences across majors were observed for MTA, NA as well as all sub-factors except SRA. However, while the highest scores for MTA were obtained by biology majors, the highest score for NA were obtained by language majors. Furthermore, within the MTA, but not the NA, sub-factors differences were observed, with psychology majors showing the highest scores for EA1, while biology majors showed the highest scores for EA2 and LMA.

In Model 1 we examined the 2-factor structure consisting of MTA and NA which was reported by Rounds and Hendel (1980) for the original MARS and assumed by Suinn and Winston (2003) for the MARS30-brief. This assumption about the factor structure of the MARS30-brief was also supported by our descriptive and normative item characteristics. MTA and NA differ not only in their mean item scores, which are lower for factors of NA (compare also Alexander and Martray, 1989), but also in their distribution characteristics. While the 15 items of MTA do not deviate from a multivariate normal distribution, thereby 
replicating findings of Hopko (2003), items of NA violate the assumption of multivariate normality. Although these results may in part have been caused by the order of items, the strength of effects suggests that another reason is more plausible. One possible explanation is the increased relevance of test situations in comparison with everyday arithmetical problems in the population tested in this study, i.e., students. This does not mean that the average scores in NA may be necessarily low and present a non-normal distribution in every population. It could be suggested that the average scores in this part of the MARS30-brief scale should be higher in populations for whom the relevance of calculation in daily living situations is higher such as by bank workers or tradesmen. This assumption was in part confirmed by our data. On the one hand, gender differences were only apparent for MTA, but not NA. On the other hand different majors showed highest values for MTA (biology) and NA (language), indicating a higher relevance of mathematical tests for science majors, but higher relevance of everyday mathematical calculations for nonscience majors. The fact that biology students show such a high degree of MTA also suggests that not all science majors can be grouped together in their evaluation of mathematics anxiety. This has, however, been done in previous comparisons of mathematics anxiety between college majors (Hamza et al., 2011). Such a grouping of all science majors may cause an over- or underestimation of mathematics anxiety differences between majors and may cost some majors (e.g., biology majors) the necessary attention they require in dealing with their mathematics anxiety.

However, using confirmatory factor analytic techniques, we could not confirm the results obtained previously with principal components analysis and a fixed number of factors. Although taking into account error covariances, the $X^{2}$ value and fit indices of Model 1 were not satisfactory. Thus, our data clearly suggest that the two global factors MTA and NA are not sufficient for describing the factor structure of the MARS30-brief, but that its factor structure has more facets.

As an alternative hypothesis (ii) one could assume that the two dimensions MTA and NA perform better describing the covariance between the more specific first order factors. Therefore, in Model 2 the MTA - NA structure was dismembered into several smaller factors in a two stage two-factor model. This model, however, had to be discarded due to negative error variances, providing support for Models 3 and 4.

Model 3 and 4 differentiate better between different aspects of mathematics anxiety. Our factors EA and LMA replicate Hopko (2003), ENA, and PA have already been reported by Bessant (1995) and SRA by Resnick et al. (1982). Our Model 3 therefore

\section{REFERENCES}

Alexander, L., and Cobb, R. (1987). Identification of the dimensions and predictors of mathematics anxiety among college students. J. Hum. Behav. Learn. 4, 25-32.

Alexander, L., and Martray, C. R. (1989). The development of an abbreviated version of the Mathematics Anxiety Rating Scale. Meas. Eval. Couns. Dev. 22, $143-150$.

Ashcraft, M. H., and Faust, M. W. (1994). Mathematics anxiety and mental arithmetic performance: an exploratory investigation. Cogn. Emot. 8, 97-125. doi: 10.1080/02699939408408931 includes all factors reported in the literature except $\mathrm{OA}$ and AA. However, OA was represented within the MARS30-brief by only 1 item (Item27), while the items of the construct AA were not originally contained in the MARS. It is to note that in contrast with findings of Bessant (1995), but replicating findings of Resnick et al. (1982), a strong association between factors ENA and PA was observed. Since Bessant (1995) forced factors ENA and PA as well as item residuals to be uncorrelated, it remains an open question, whether this association is generally high or only in our specific population.

However, contrary to the literature, the best model fit was obtained when further splitting EA into two factors capturing different aspects of EA, i.e., EA proper (taking and examination) as opposed to EA2 (just thinking about an examination). These two factors particularly seemed to induce different levels of anxiety across different majors with psychology majors showing particularly high values on EA1, but low values on all other aspects of mathematics anxiety. Furthermore, the correlation between EA1 and ENA was almost 0, whereas the correlation between EA2 and ENA was of moderate strength, suggesting different qualities of these two factors. We do note, however, that the results on differences between college majors need to be interpreted with care, since measurement invariance could not be established for these groups due to small sample sizes. Since Model fit of Model 3 is also acceptable and Model 3 is more parsimonious, Model 3 is probably the most practical model for research questions not evaluating differences between college majors.

In summary, the present findings on mathematics anxiety do not support the view that it can be reduced to MTA as has been suggested by Plake and Parker (1982) and Hopko (2003). Dew and Galassi (1983) and Dew et al. (1984) found that mathematics anxiety measures are more highly related to each other than to measures of test anxiety and therefore still reflect different aspects of personality. For a successful career it could rather be important to reduce SRA and PA to a reasonable and productive value. Through such an approach of differential diagnosis, intervention can target especially those constructs with high scores.

\section{ACKNOWLEDGMENTS}

We very much wish to acknowledge Richard Suinn for providing the MARS30-brief and all participants for their time and willingness to contribute to this study. Furthermore, we thank Ulrike Kipman for statistical advice.

Ashcraft, M. H., and Kirk, E. P. (2001). The relationships among working memory, mathematics anxiety, and performance. J. Exp. Psychol. Gen. 130, 224-237. doi: 10.1037/0096-3445.130.2.224

Baloğlu, M., and Koçak, R. (2006). A multivariate investigation of the differences in mathematics anxiety. Pers. Individ. Dif. 40, 1325-1335. doi: 10.1016/j.paid.2005.10.009

Bentler, P. M., and Dudgeon, P. (1996). Covariance analysis: statistical practice, theory, and directions. Annu. Rev. Psychol. 47, 563-592. doi: 10.1146/annurev.psych.47.1.563

Bessant, K. C. (1995). Factors associated with types of mathematics anxiety in college students. J. Res. Math. Educ. 26, 327-345. doi: 10.2307/749478 
Brush, L. R. (1978). A validation study of the Mathematics Anxiety Rating Scale (MARS). Educ. Psychol. Meas. 38, 485-490. doi: 10.1177/0013164478038 00236

Chen, F. F. (2007). Sensitivity of goodness of fit indexes to lack of measurement invariance. Struct. Equ. Model. A Multidiscip. J. 14, 464-504. doi: 10.1080/10705510701301834

Devine, A., Fawcett, K., Szucs, D., and Dowker, A. (2012). Gender differences in mathematics anxiety and relation to mathematics performance while controlling for test anxiety. Behav. Brain Funct. 8, 33. doi: 10.1186/17449081-8-33

Dew, K. H., and Galassi, J. P. (1983). Mathematics anxiety: some basic issues. J. Couns. Psychol. 30, 443-446. doi: 10.1007/s11136-010-9623-7

Dew, K. H., Galassi, J. P., and Galassi, M. D. (1984). Mathematics anxiety: relation with situational test anxiety, performance, physiological arousal, and mathematics avoidance behavior. J. Couns. Psychol. 31, 580-583. doi: 10.1037/0022-0167.31.4.580

Evans, J. (2000). Adults' Mathematical Thinking and Emotions: A Study of Numerate Practice. Hove: Psychology Press.

Fabrigar, L. R., Wegener, D. T., MacCallum, R. C., and Straham, E. J. (1999). Evaluating the use of exploratory factor analysis in psychological research. Psychol. Methods 4, 272-299. doi: 10.1037/1082-989X.4.3.272

Ferguson, R. D. (1986). Abstraction anxiety: a factor of mathematics anxiety. J. Res. Math. Educ. 17, 145-150. doi: 10.2307/749260

Fujii, Y. (1994). A study on Mathematics Anxiety Rating Scale (MARS). Jpn. J. Educ. Psychol. 42, 448-454. doi: 10.5926/jjep1953.42.4_448

Hamza, E. A., Helal, A., and Hagstrom, F. (2011). Math anxiety in college students across majors. Int. J. Arts Sci. 4, 211-221.

Hopko, D. R. (2003). Confirmatory factor analysis of the mathematics anxiety rating scale-revised. Educ. Psychol. Meas. 63, 336-351. doi: 10.1177/0013164402251041

Hopko, D. R., Mahadevan, R., Bare, R. L., and Hunt, M. K. (2003). The Abbreviated Mathematics Anxiety Scale (AMAS): construction, validity, and reliability. Assessment 10, 178-182. doi: 10.1177/1073191103010002008

Hopko, D. R., McNeil, D. W., Zvolensky, M. J., and Eifert, G. H. (2001). The relation between anxiety and skill in performance-based anxiety disorders: a behavioral formulation of social phobia. Behav. Ther. 32, 185-207. doi: 10.1016/S0005-7894(01)80052-6

Jänen, I., Gölitz, D., Roick, T., and Hasselhorn, M. (2006). "Gibt es geschlechtsunterschiede im ausmaß und der situationsspezifik von mathematikangst? [Are there gender differences in the extent and situation specificity of mathematics anxiety?]," in Poster at the 48th Tagung Experimentell Arbeitender Psychologen (TeaP), 26-29. April 2006, Mainz.

Levitt, E. E., and Hutton, L. H. (1984). A psychometric assessment of the Mathematics Anxiety Rating Scale. Int. Rev. Appl. Psychol. 33, 233-242. doi: 10.1111/j.1464-0597.1984.tb01431.x

Marsh, H. W., and Hocevar, D. (1985). Application of Confirmatory Factor Analysis to the study of Self-Concept: first and higher order factor models and their invariance across groups. Psychol. Bull. 97, 562-582. doi: 10.1037/00332909.97.3.562

Morris, L. W., Kellaway, D. S., and Smith, D. H. (1978). Mathematics anxiety rating scale: predicting anxiety experience and academic performance in two groups of students. J. Educ. Psychol. 70, 589-594. doi: 10.1037/0022-0663.70. 4.589

Plake, B. S., and Parker, C. S. (1982). The development and validation of a revised version of the Mathematics Anxiety Rating Scale. Educ. Psychol. Meas. 42, 551-557. doi: 10.1177/001316448204200218

Preston, P. A. (1986). Math Anxiety: Relationship with Sex, College Major, Mathematics Background, Mathematics Achievement, Mathematics Performance, Mathematics Avoidance, Self-Rating of Math-Ematics Ability, and Self-Rating of Mathematics Anxiety as Measured by the Revised Mathematics Anxiety Rating Scale (RMARS). Doctoral dissertation, University of Tennessee, Knoxville, TN, 47, 2494A.

Resnick, H., Viehe, J., and Segal, S. (1982). Is mathematics anxiety a local phenomenon? A study of prevalence and dimensionality. J. Couns. Psychol. 29, 39-47. doi: 10.1037/0022-0167.29.1.39

Richardson, F. C., and Suinn, R. M. (1972). The mathematics anxiety rating scale: psychometric data. J. Couns. Psychol. 19, 551-554. doi: 10.1037/h0033456

Richardson, F. C., and Suinn, R. M. (1973). A comparision of traditional systematic desensitization, accelerated massed desensitization, and anxiety management training in the treatment of mathematics anxiety. Behav. Ther. 4, 212-218. doi: 10.1016/S0005-7894(73)80030-9

Rounds, J. B., and Hendel, D. D. (1980). Measurement and dimensionality of mathematics anxiety. J. Couns. Psychol. 27, 138-149. doi: 10.1037/00220167.27.2.138

Schumacker, R. E., and Lomax, R. G. (2004). A Beginners' Guide to Structural Equation Modelling, 2nd Edn. London: Lawrence Erlbaum Associates.

Suinn, R. M., Edie, C. A., Nicoleti, J., and Spenelli, P. R. (1972). The MARS, a measure of mathematics anxiety: psychometric data. J. Clin. Psychol. 28, 373-375. doi: 10.1002/1097-4679(197207)28:3+<373::AIDJCLP2270280320> 3.0.CO;2-C

Suinn, R. M., and Winston, E. H. (2003). The Mathematics Anxiety Rating Scale, a brief version: psychometric data. Psychol. Rep. 92, 167-173. doi: 10.2466/pr0.2003.92.1.167

Wilder, S. (2012). Gender Differences in Factors Pertaining to Math Anxiety Among College Students. Electronic Thesis or Dissertation, Doctor of Philosophy, University of Akron, Akron. Available at: https://etd.ohiolink.edu/

Conflict of Interest Statement: The authors declare that the research was conducted in the absence of any commercial or financial relationships that could be construed as a potential conflict of interest.

The reviewer $\mathrm{CP}$ and handling Editor declared a current collaboration and the handling Editor states that the process nevertheless met the standards of a fair and objective review.

Copyright (c) 2016 Pletzer, Wood, Scherndl, Kerschbaum and Nuerk. This is an open-access article distributed under the terms of the Creative Commons Attribution License (CC BY). The use, distribution or reproduction in other forums is permitted, provided the original author(s) or licensor are credited and that the original publication in this journal is cited, in accordance with accepted academic practice. No use, distribution or reproduction is permitted which does not comply with these terms. 